\title{
Post-hemorrhagic shock mesenteric lymph is an important contributor to cardiac dysfunction following hemorrhagic shock ${ }^{1}$
}

\author{
Hui-bo Du', Si-hai Wang' ${ }^{\text {II }}$ Zi-gang Zhao'II, Chun-yu Niu ${ }^{\text {III }}$ \\ DOI: http://dx.doi.org/10.1590/S0102-865020150060000010
}

'Bachelor, Experimentalist, Institute of Microcirculation, Hebei North University, Zhangjiakou, China. Acquisition of data, manuscript writing, critical revision.

"Master, Associate Chief Physician, Institute of Microcirculation, Hebei North University, Zhangjiakou, China. Acquisition of data.

IIIPhD, Full Professor, Institute of Microcirculation, Hebei North University, Zhangjiakou, China. Design of the study, critical revision.

\begin{abstract}
PURPOSE: To evaluate whether post-hemorrhagic shock mesenteric lymph (PSML) is involved in cardiac dysfunction induced by hemorrhagic shock.

METHODS: The hemorrhagic shock model $(40 \pm 2 \mathrm{mmHg}, 3 \mathrm{~h})$ was established in rats of the shock and shock+drainage groups; and PSML drainage was performed from hypotension $1-3 \mathrm{~h}$ in the shock+drainage rats. Then, the isolated hearts were obtained from the rats for the examination of cardiac function with Langendorff system. Subsequently, the isolated hearts were obtained from normal rats and perfused with PSML or Krebs-Henseleit solution, and the changes of cardiac function were observed.

RESULTS: The left ventricular systolic pressure (LVSP) and the maximal rates of LV developed pressure (LVDP) rise and fall ( $\pm \mathrm{dP} /$ $\left.\mathrm{d} t_{\max }\right)$ in the shock and shock+drainage groups were lower than that of the sham group; otherwise, these indices in the shock+drainage group were higher compared to the shock group. In addition, after isolated hearts obtained from normal rats perfusing with PSML, these cardiac function indices were gradual decline along with the extension of time, such as heart rate, $\mathrm{LVSP}, \pm \mathrm{dP} / \mathrm{d} t_{\max }$, etc.
\end{abstract}

CONCLUSION: Post-hemorrhagic shock mesenteric lymph is an important contributor to cardiac dysfunction following hemorrhagic shock.

Key words: Shock, Hemorrhagic. Lymph. Heart Failure, Systolic. Rats. 


\section{Introduction}

Cardiac pump function plays an important role in maintaining blood flow dynamics and ensuring tissue perfusion. Cardiac systolic dysfunction is a key link of aggravating microcirculation disorder and causing other organ injuries following trauma, hemorrhagic shock, endotoxic shock, and sepsis, etc., which is a major cause of death $^{1-3}$. Therefore, there is important theoretical significance and practical value in exploring the mechanism of the cardiac dysfunction for finding new intervention measures to prevent and control the severe shock and multiple organ dysfunction syndrome (MODS) or multiple organ failure. Increasing studies have demonstrated that the post-hemorrhagic shock mesenteric lymph (PSML) return has been implicated in the pathogenesis of MODS following severe hemorrhagic shock ${ }^{4-6}$. Our previous studies showed that blockage of PSML return by mesenteric lymph duct ligation (MLDL) could alleviate the myocardium injury in rats following hemorrhagic shock with fluid resuscitation, and its mechanism was related to decreasing free radical injury and inflammation ${ }^{7}$. Recently, Sambol et al. ${ }^{8}$ found that the MLDL could prevent hemorrhagic shock-induced cardiac contractile dysfunction, and PSML intravenous infusion into naïve rats induced myocardial contractile dysfunction 9 . Thus, the current study further tests the hypothesis that PSML is involved in cardiac dysfunction after hemorrhagic shock.

\section{Methods}

All animal procedures were reviewed and approved by the Institutional Animal Care and Use Committee of Hebei North University and conformed to National Institutes of Health guidelines. All efforts were made to minimize suffering of animals.

Twenty-eight adult male Wistar rats, weighing 230-270g, were purchased from the Laboratory Animal Breeding Center of the Chinese Academy of Medical Sciences (Beijing, China). Before the experimentation, the rats were fasted and allowed free access to water for $12 \mathrm{~h}$.

\section{Hemorrhagic shock model and PSML drainage}

The eighteen rats were anesthetized with $1 \%$ pentobarbital sodium $(50 \mathrm{mg} / \mathrm{kg})$. Under aseptic conditions, all of the rats received femoral operations to separate the right femoral vein and bilateral femoral arteries for anticoagulation, hemorrhage, and monitoring of mean arterial pressure (MAP) as previously reported ${ }^{10}$. Afterwards, the rats received abdominal operation to separate the mesenteric lymph duct from the surrounding connective tissues for the drainage of PSML. After an equilibrium period of $30 \mathrm{~min}$, the rats were randomly divided into the sham group, shock group (hemorrhagic shock model), and shock+drainage group (hemorrhagic shock plus mesenteric lymph drainage), $n=6$ rats in each group. In the shock and shock+drainage groups, the hemorrhagic shock model was established with the method of hemorrhage through the left femoral artery, and the MAP was maintained at a level of 40 $\mathrm{mmHg}$ for $3 \mathrm{~h}$ by withdrawing or reinfusing shed blood as needed. In the shock+drainage group, the mesenteric lymph drainage was performed from $1 \mathrm{~h}$ to $3 \mathrm{~h}$ of hypotension as previously described ${ }^{10}$, the PSML were centrifuged at $315 \mathrm{~g}$ at $0-4^{\circ} \mathrm{C}$ for $5 \mathrm{~min}$ to remove all cellular elements and flash frozen at $-75^{\circ} \mathrm{C}$ in refrigerator (Thermo Electron, Waltham, MA) for the further experimentation. In the sham group, the rats were anesthetized and received the same operation as described above, but did not undergo hemorrhage and PSML drainage.

\section{Measurement of cardiac function of isolated heart from hemorrhagic shocked rats}

After hypotension for $3 \mathrm{~h}$ in the shock and shock+drainage groups or corresponding time in the sham group, under deeply anesthetic conditions, the hearts were quickly excised from rats, and then immersed in $\mathrm{Ca}^{2+}$-free Krebs-Henseleit solution contained (in $\mathrm{mmol} / \mathrm{L}$ ) $118.0 \mathrm{NaCl}, 4.7 \mathrm{KCl}, 1.2 \mathrm{MgCl}_{2}, 25.0 \mathrm{NaHCO}_{3}, 1.2$ $\mathrm{KH}_{2} \mathrm{PO}_{4}, 11.0$ glucose, $2.5 \mathrm{CaCl}_{2}, \mathrm{pH} 7.3-7.4$; which was oxygenated by bubbling with $95 \% \mathrm{O}_{2}$ and $5 \% \mathrm{CO}_{2}$, at temperature of $0-4{ }^{\circ} \mathrm{C}$.

Subsequently, the blood in ventricle was discharged through ventricular extrusion. And then, the ascending aorta was cannulated, and the catheter was attached to a Langendorff system (ADInstruments, Bella Vista NSW, Australia). The hearts were perfused in a retrograde fashion with Krebs-Henseleit solution, which was oxygenated by bubbling with $95 \% \mathrm{O}_{2}$ and $5 \% \mathrm{CO}_{2}$. The whole system was water jacketed and maintained at $37^{\circ} \mathrm{C}$.

After a 15-min stabilization period, these indices of isolated heart function, including the left ventricular systolic pressure (LVSP), left ventricular end diastolic pressure (LVEDP), heart rate (HR), and the maximum rates of left ventricular developed pressure (LVDP) rise $\left(+\mathrm{dP} / \mathrm{d} t_{\max }\right)$ and fall $\left(-\mathrm{dP} / \mathrm{d} t_{\max }\right)$, were assessed by measuring the intraventricular pressure with a fluidfilled balloon (polyethylene film) that had been inserted into the left ventricle via the mitral valve from the left atrium as previously described ${ }^{8,9,11}$. This balloon was connected to a pressure transducer (ADInstruments), LVDP was calculated as the difference between the peak LVSP and LVEDP. And all parameters were analyzed offline using PowerLab software (ADInstruments). 


\section{Experiments cardiac function of isolated heart from normal rats perfused by $P S M L$}

The ten normal male rats were anesthetized and the hearts were removed and perfused with Krebs-Henseleit buffer as described above. After an equilibrium period of $20 \mathrm{~min}$, the isolated hearts were randomly divided into the control group and PSML group, $n=5$ hearts in each group. In the control group, the isolated hearts were perfused with Krebs-Henseleit buffer. In the PSML group, the isolated hearts were perfused with PSML of $4 \%$ $(\mathrm{V}: \mathrm{V})$, which harvested from the hemorrhagic shocked rats in the above experiment. According to the method as described above, the isolated heart function was assessed at $0,2,5,10,15$, and 20 min after perfusion with Krebs-Henseleit buffer or PSML.

\section{Statistical analysis}

Data are presented as the mean \pm SD and were analyzed using SPSS version 16.0 software. One-way analysis of variance was used to identify differences within three groups, followed by Student-Newman-Keuls $q$ test was used to identify differences between groups. In addition, the IndependentSamples $t$ test was used to identify differences between the control and PSML groups. Values of $p<0.05$ were considered statistically significant.

\section{Results}

\section{Role of PSML drainage on cardiac function of isolated hearts obtained from hemorrhagic shocked rats}

Figure 1 showed that the LVSP and $\pm \mathrm{dP} / \mathrm{d} t_{\max }$ of isolated hearts obtained from the shock and shock+drainage groups were significantly decreased, and HR was increased compared to the sham group, respectively $(\mathrm{p}<0.05)$. Meanwhile, the LVSP and $\pm \mathrm{dP} / \mathrm{d} t_{\max }$ in the shock + drainage group were increased than that in the shock group $(\mathrm{p}<0.05)$. Moreover, there was no statistics difference in LVEDP among three groups ( $p>0.05)$.
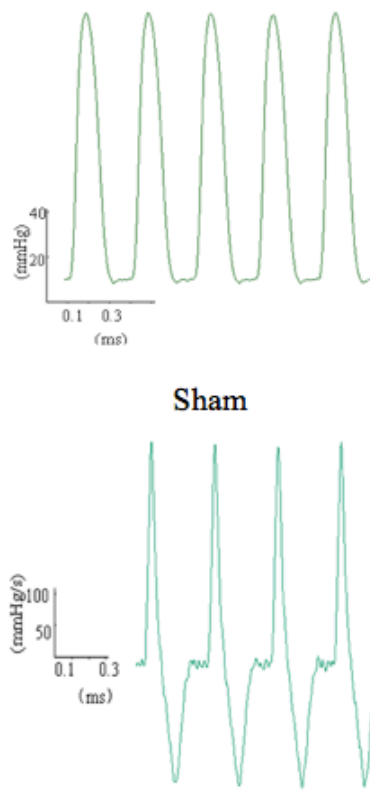

Sham

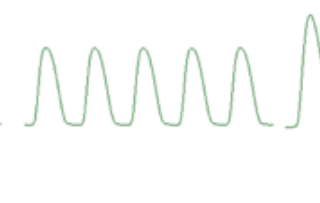

Shock

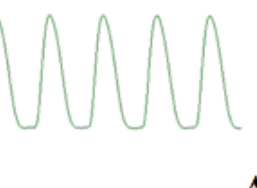

$\mathbf{A}$

Shock+drainalge

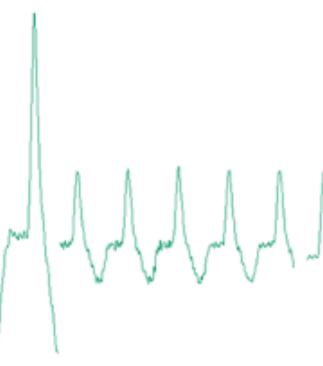

.

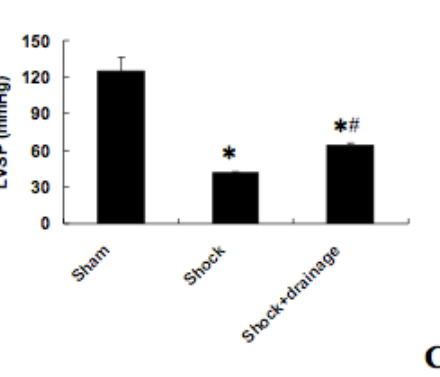

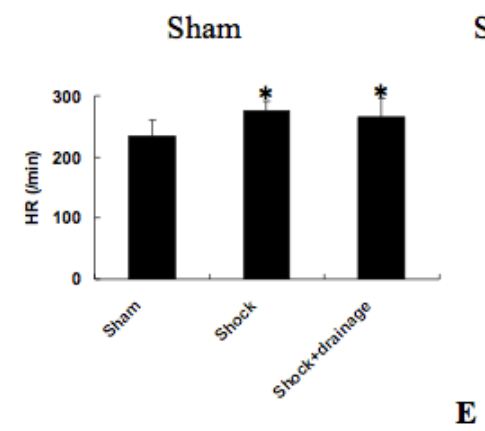

Shock
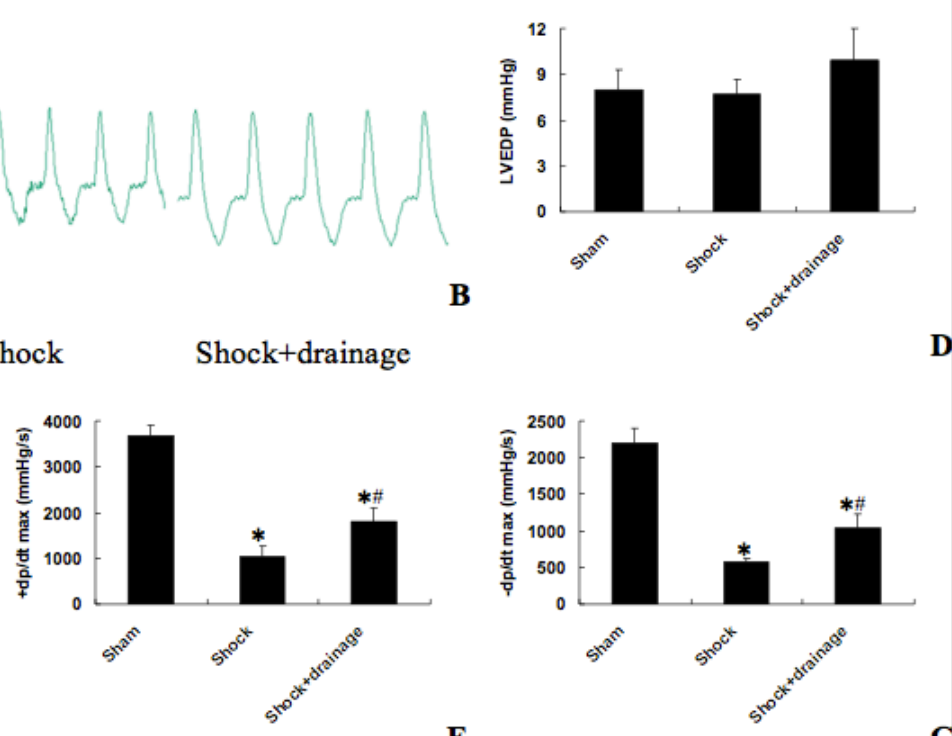

$\mathbf{F}$

G

FIGURE 1 - Role of post-hemorrhagic shock mesenteric lymph (PSML) drainage on function of isolated heart obtained from hemorrhagic shock rats (mean $\pm \mathrm{SD}, \mathrm{n}=6$ ). A) Image of arterial blood pressure in isolated hearts. B) Image of $\pm \mathrm{dP} / \mathrm{d} t_{\max }$ in isolated hearts. C) Change of left ventricular systolic pressure (LVSP) in isolated hearts. D) Change of left ventricular end diastolic pressure (LVEDP) in isolated hearts. E) Change of heart rate (HR) in isolated hearts. F) Change of $+\mathrm{dP} / \mathrm{d} t_{\max }$ in isolated hearts. G) Change of $-\mathrm{dP} / \mathrm{d} t_{\max }$ in isolated hearts. ${ }^{*} \mathrm{p}<0.05$ vs. the sham group; ${ }^{*} \mathrm{p}<0.05$ vs. the shock group. 
Role of PSML perfusion on cardiac function of isolated hearts obtained from normal rats

Figure 2 showed the representative images of isolated heart obtained from normal rats following perfusion with KrebsHenseleit buffer or PSML. There were no significantly changes in LVSP, LVEDP, HR, and $\pm \mathrm{dP} / \mathrm{d} t_{\text {max }}$ levels in the control group during perfusion with Krebs-Henseleit buffer ( $p>0.05$ ). Meanwhile, there were no statistical differences in cardiac function indices before perfusion between the control and PSML groups $(\mathrm{p}>0.05)$. In addition, in the PSML group, there were downward trend in HR, LVSP, and $\pm \mathrm{dP} / \mathrm{d} t_{\text {max }}$, upward trend in LVEDP, and there were significantly differences at multiple times after perfusion with PSML compare to $0 \min (p<0.05)$. Meanwhile, the LVEDP at 10 and $15 \mathrm{~min}$ in the PSML group were higher, the $\mathrm{HR}$ and $-\mathrm{dP} / \mathrm{d} t_{\text {max }}$ at $5,10,15$, and $20 \mathrm{~min}$, the + $\mathrm{dP} / \mathrm{d} t_{\text {max }}$ at 15 and 20 min were lower compared with the control group, respectively $(\mathrm{p}<0.05)$ (Figure 3 ).
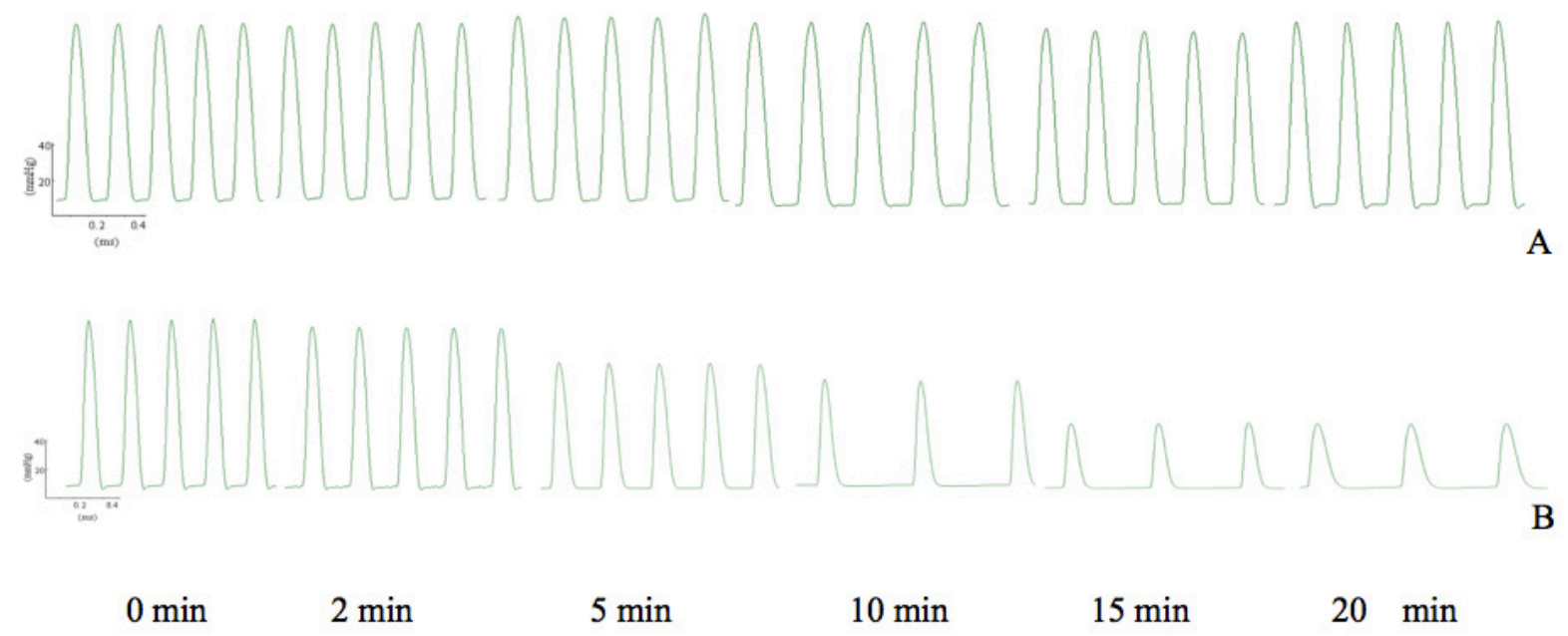

FIGURE 2 - Images of post-hemorrhagic shock mesenteric lymph (PSML) perfusion on function of isolated hearts obtained from normal rats. A) Control group. B) PSML group.
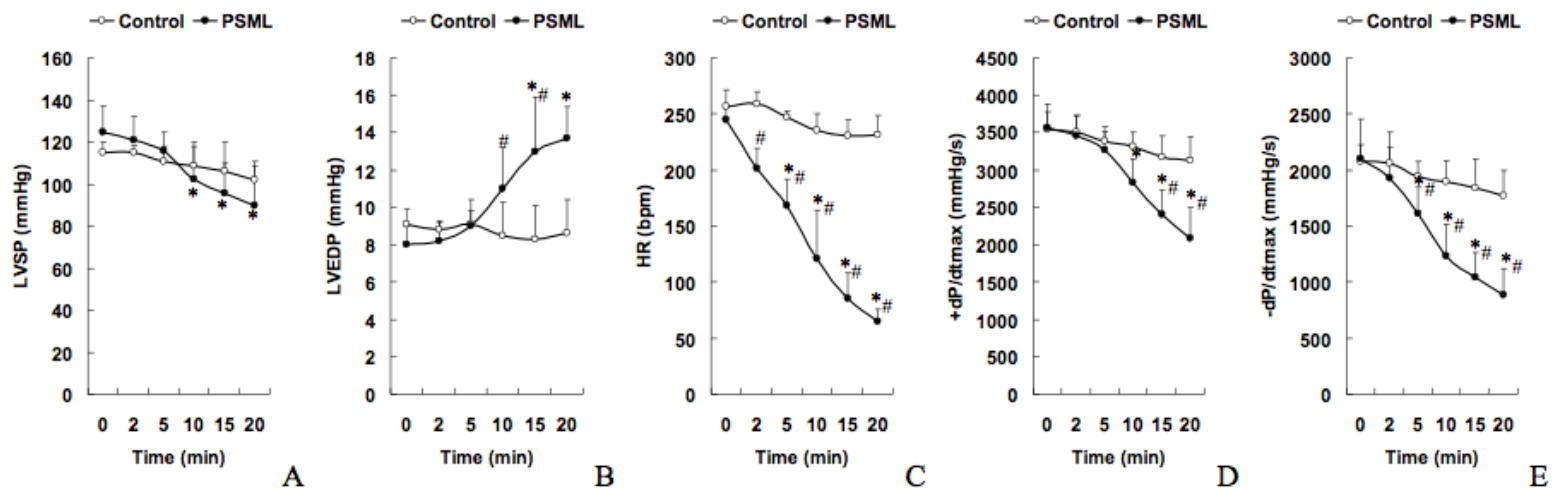

FIGURE 3 - Role of post-hemorrhagic shock mesenteric lymph (PSML) perfusion on function of isolated hearts obtained from normal rats (mean $\pm \mathrm{SD}, \mathrm{n}=5$ ). A) Change of left ventricular systolic pressure (LVSP) in isolated hearts. B) Change of left ventricular end diastolic pressure (LVEDP) in isolated hearts. C) Change of heart rate (HR) in isolated hearts. D) Change of $+\mathrm{dP} / \mathrm{d} t_{\max }$ in isolated hearts. E) Change of $-\mathrm{dP} / \mathrm{d} t_{\max }$ in isolated hearts. ${ }^{*} \mathrm{p}<0.05 v s$. the value at $0 \mathrm{~min}$ in the same group; ${ }^{*} \mathrm{p}<0.05$ $v s$. the control group at the same time.

\section{Discussion}

Cardiac dysfunction following hemorrhagic shock has been well described in the literature ${ }^{12-14}$. However, the detailed mechanisms remain further research. Based on our experimental studies implicating gut-derived factors carried in the mesenteric lymph as contributing factors to myocardial damage after hemorrhagic shock, we investigated the effects of PSML on cardiac function after hemorrhagic shock, and found that PSML return is centrally involved in hemorrhagic shock-induced cardiac dysfunction. 
The intestinal lymph pathway plays an important role in organ injuries in severe pathological conditions, such as hemorrhagic and traumatic shock and acute pancreatitis ${ }^{5,15}$. The report form Sambol et al. ${ }^{8}$ found that MLDL could prevent trauma/ hemorrhage shock-induced increases in LVDP and $\pm \mathrm{dP} / \mathrm{d} t_{\max }$ of isolated hearts form trauma/hemorrhage shocked rats. In the present study, we found that PSML drainage also could increase the LVSP and $\pm \mathrm{dP} / \mathrm{d} t_{\max }$, which was consistent with the role of MLDL on cardiac function. So that, the current results further suggest that the PSML return is an important factor for cardiac dysfunction subjected to hemorrhagic shock.

In order to further investigate the role of PSML on cardiac dysfunction in vitro, we isolated hearts from normal rats, and perfused the isolated hearts with 4\% PMSL, observed the changes of cardiac contractile function. The results showed that perfusion with PSML induced a gradual decline in the LVSP, HR, and $\pm \mathrm{dP} /$ $\mathrm{d} t_{\max }$ levels, and an upward trend in LVEDP. Meanwhile, there were significantly differences at multiple times between the control and PSML perfusion. The roles of PSML perfusion decreasing LVSP and $\pm \mathrm{dP} / \mathrm{d} t_{\text {max }}$ levels were similar with hemorrhagic shock induced decreases in LVDP and $\pm \mathrm{dP} / \mathrm{d} t_{\text {max }}$ in vivo, which reported by previous literature ${ }^{8}$. Therefore, these findings further indicated that PSML return play a key role in the pathogenesis of abnormal cardiac function following hemorrhagic shock.

At present, hemorrhagic shock-induced myocardial contractile dysfunction is related to the inflammatory response, and the myocardial depression is mediated by proinflammatory mediators ${ }^{16-18}$. Based on previous study implicating gut-derived factors carried in the mesenteric $\operatorname{lymph}^{19,20}$, as well as the reduction of MLDL on inflammatory state in myocardium ${ }^{7}$, we considered that gut-derived inflammatory factors might be involved in PSML-induced cardiac dysfunction. However, the detailed components resulted in the cardiac contractile dysfunction contained in the PSML should be investigated in the future.

It should be pointed out that the HR of isolated hearts from hemorrhagic shocked rats was significantly increased than that the sham group, which is inconsistent with the role of PSML perfusion decreasing the HR of isolated hearts from normal rats. Its reason might be related to the compensatory induced by acute hemorrhage and the short observation time. Hence, further research is needed in the future. In addition, the results of the present study showed that there were no significant changes in the control isolated hearts during the perfused period, indicating that the Langendorff system employed in this research is stable and applicable.

\section{Conclusions}

Post-hemorrhagic shock mesenteric lymph (PSML) drainage improved the cardiac function of isolated hearts obtained from rats following hemorrhagic shock, and PSML perfusion caused cardiac contractile dysfunction of isolated hearts obtained from normal rats. These results indicate that PSML is an important contributor to cardiac dysfunction after hemorrhagic shock.

\section{References}

1. Chatpun S, Cabrales P. Cardiac systolic function recovery after hemorrhage determines survivability during shock. J Trauma. 2011 Apr;70(4):787-93. doi: 10.1097/TA.0b013e3181e7954f.

2. Zaky A, Deem S, Bendjelid K, Treggiari MM. Characterization of cardiac dysfunction in sepsis: an ongoing challenge. Shock. 2014 Jan;41(1):12-24. doi: 10.1097/SHK.0000000000000065.

3. Piquereau J, Godin R, Deschênes S, Bessi VL, Mofarrahi M, Hussain SN, Burelle Y. Protective role of PARK2/Parkin in sepsis-induced cardiac contractile and mitochondrial dysfunction. Autophagy. 2013 Nov;9(11):1837-51. doi: 10.4161/auto.26502.

4. Deitch EA. Gut-origin sepsis: evolution of a concept. Surgeon. 2012 Dec;10(6):350-6. doi: 10.1016/j.surge.2012.03.003

5. Deitch EA. Gut lymph and lymphatics: a source of factors leading to organ injury and dysfunction. Ann NYAcad Sci. 2010 Oct;1207(Suppl 1):E103-11. doi: 10.1111/j.1749-6632.2010.05713.x.

6. Cai B, Deitch EA, Ulloa L. Novel insights for systemic inflammation in sepsis and hemorrhage. Mediators Inflamm. 2010 Jun;2010:642462. doi: 10.1155/2010/642462.

7. Zhao ZG, Niu CY, Chen RH, Zhang YP, Zhang J, Liu YK, Li JC. Effect of intestinal lymphatic pathway on free radical and inflammatory mediator of myocardium in shock rats. Zhongguo Ying Yong Sheng Li Xue Za Zhi. 2007 Nov;23(4):385-9. doi: 10.3969/j.issn.1000-6834.2007.04.001.

8. Sambol JT, Lee MA, Caputo FJ, Kawai K, Badami C, Kawai T, Deitch EA, Yatani A. Mesenteric lymph duct ligation prevents trauma/hemorrhage shock-induced cardiac contractile dysfunction. J Appl Physiol (1985). 2009 Jan;106(1):57-65. doi: 10.1152/ japplphysiol.90937.2008.

9. Sambol JT, Lee MA, Jiang M, Dosi G, Dong W, Deitch EA, Yatani A. Mesenteric lymph from rats with trauma-hemorrhagic shock causes abnormal cardiac myocyte function and induces myocardial contractile dysfunction. J Appl Physiol (1985). 2011 Sep;111(3):799807. doi: 10.1152/japplphysiol.00100.2011.

10. Zhao ZG, Niu CY, Wei YL, Zhang YP, Si YH, Zhang J. Mesenteric lymph return is an important contributor to vascular hyporeactivity and calcium desensitization after hemorrhagic shock. Shock. 2012 Aug;38(2):186-95. doi: 10.1097/SHK.0b013e31825f1c9b.

11. Sutherland FJ, Hearse DJ. The isolated blood and perfusion fluid perfused heart. Pharmacol Res. 2000 Jun;41(6):613-27. doi: 10.1006/phrs.1999.0653.

12. Yu HP, Yang S, Choudhry MA, Hsieh YC, Bland KI, Chaudry IH. Mechanism responsible for the salutary effects of flutamide on cardiac performance after trauma-hemorrhagic shock: Upregulation of cardiomyocyte estrogen receptors. Surgery. 2005 Jul;138(1):8592. doi: http://dx.doi.org/10.1016/j.surg.2005.03.006.

13. Zou L, Yang S, Champattanachai V, Hu S, Chaudry IH, Marchase $\mathrm{RB}$, Chatham JC. Glucosamine improves cardiac function following trauma-hemorrhage by increased protein O-GlcNAcylation and attenuation of NF-\{kappa\}B signaling. Am J Physiol 
$\mathrm{Du} \mathrm{H}$ et al.

Heart Circ Physiol. 2009 Feb;296(2):H515-23. doi: 10.1152/ ajpheart.01025.2008.

14. Kim H, Chen J, Zinn KR, Hubbard WJ, Fineberg NS, Chaudry IH. Single photon emission computed tomography demonstrated efficacy of $17 \beta$-estradiol therapy in male rats after trauma-hemorrhage and extended hypotension. J Trauma. 2010 Nov;69(5):1266-73. doi: 10.1097/TA.0b013e3181dbb0b2.

15. Caputo FJ, Rupani B, Watkins AC, Barlos D, Vega D, Senthil M, Deitch EA. Pancreatic duct ligation abrogates the trauma hemorrhage-induced gut barrier failure and the subsequent production of biologically active intestinal lymph. Shock. 2007 Oct;28(4):441-6. doi: 10.1097/shk.0b013e31804858f2.

16. Yang S, Hu S, Hsieh YC, Choudhry MA, Rue LW 3rd, Bland KI, Chaudry IH. Mechanism of IL-6-mediated cardiac dysfunction following trauma-hemorrhage. J Mol Cell Cardiol. 2006 Apr;40(4):570-9. doi: 10.1016/j.yjmcc.2006.01.008.

17. Meng X, Ao L, Song Y, Raeburn CD, Fullerton DA, Harken AH. Signaling for myocardial depression in hemorrhagic shock: roles of Toll-like receptor 4 and p55 TNF-alpha receptor. Am J Physiol Regul Integr Comp Physiol. 2005 Mar;288(3):R600-6. doi: 10.1152/ ajpregu.00182.2004.

18. Chen D, Assad-Kottner C, Orrego C, Torre-Amione G. Cytokines and acute heart failure. Crit Care Med. 2008 Jan;36(1 Suppl):S9-16. doi: 10.1097/01.CCM.0000297160.48694.90.

19. Deitch EA, Xu D, Kaise VL. Role of the gut in the development of injury- and shock induced SIRS and MODS: the gut-lymph hypothesis, a review. Front Biosci. 2006 Jan;11:520-8. PMID: 16146750 .
20. Niu CY, Hou YL, Zhao ZG, Zhang YF, Ji JJ, Qiao HX, Zhang J, Yao YM. Role of intestinal lymphatic pathway in pathogenesis of intestine-derived bacteria/endotoxin translocation in rats in shock. Zhongguo Wei Zhong Bing Ji Jiu Yi Xue. 2007 May;19(5):266-9. PMID: 17490562.

\section{Correspondence:}

Chun-yu Niu and Zi-gang Zhao

Institute of Microcirculation, Hebei North University

Zhangjiakou Hebei, 075000, China

Phones: (86)0313-4029168/0313-4029223

ncylxf@126.com

lymphatics@126.com

zzghyl@126.com

Received: Feb 14, 2015

Review: Apr 13, 2015

Accepted: May 14, 2015

Conflict of interest: none

Financial sources: Hebei Province (2011-04) and Hebei North University (CXRC1314)

${ }^{1}$ Research performed at Institute of Microcirculation, Hebei North University, Zhangjiakou Hebei, China. 